\title{
Multispectral imaging of normal bladder mucous membrane and clinical stage of bladder tumor
}

\author{
Xiao-feng Yang ${ }^{{ }^{*}}$, Xiao-jing Zhang ${ }^{\mathrm{a}}$, Sen Yang ${ }^{\mathrm{a}}$, Zi-qiang Zhang $^{\mathrm{a}}$, Wei Zhang ${ }^{\mathrm{a}}$, Dong-wen Wang ${ }^{\mathrm{a}}$ \\ ${ }^{a}$ Department of Urology, First Affiliated Hospital of Shanxi Medical University, Taiyuan 030001, China.
}

\begin{abstract}
Objective: To explore the imaging characteristics and clinical significance of multispectral cystoscopy. Methods: A total of 14 patients with benign prostatic hyperplasia and 16 patients with bladder tumor were enrolled from May 2011 to May 2013; five patients with normal bladder were enrolled as a control group. After the patients were anesthetized, a resectoscope was inserted into the bladder through the urethra. White, ultraviolet (UV), blue, green, red, and near-infrared lights were used for cystoscopy.

Results: When UV and near-infrared lights were used to irradiate the bladders, there were no images on the monitor. However, the bladder mucosa and submucosa blood vessels showed clearly and constituted a threedimensional mesh when using green light; the density of blood vessels was increased but was less clear when using blue light $(\mathrm{p}<0.05)$, and the mucosal surface was covered in red when using red light. After transurethral resection of bladder tumor (TURBt) for T1 stage tumors was performed, the superficial muscle layer with integrity structure could be observed under white light, but blood vessels did not appear when using green light. Ta stage tumors were shown to be connected to the mucosal vasculature by only one small blood vessel when viewed under green light. Blood vessels were normal when usingwhite light. Structural disorders seen under green light should be highly suspected as Tis-stage tumors.

Conclusion: Tumor blood vessels and bladder mucosal vasculature can be shown by green light without the use of photosensitizers or fluorescent dyes. Hemoglobin absorbs light at different wavelengths. Thus, according to the principles of imaging, small tumors and invasive tumor depth can be identified under green light. cystoscopy.

Keywords: Bladder mucosa; Bladder tumor; Blood vessel; Optical imaging; cystoscopy.
\end{abstract}

\section{INTRODUCTION}

According to the literature, about 357,000 cases of new bladder cancer (BC) are diagnosed each year ${ }^{[1]}$. Approximately $75-85 \%$ of all patients with $\mathrm{BC}$ have disease that is confined to the mucosa or submucosa. This group of tumors is referred to as non-muscle-invasive or superficial $\mathrm{BC}$, in contrast to muscle-invasive disease staged T2-T4. Most patients showed non-muscle-invasive bladder cancer (NMIBC) and are at risk of recurrence. Because of this high recurrence rate, patients with NMIBC require lifelong follow-up with cystoscopy and cytology are needed ${ }^{[2]}$. As a critical component of BC evaluation, the quality of cystoscopy and tumor resection may directly influence the cancer's outcome. White light cystoscopy (WLC), the traditional standard diagnosis for

\footnotetext{
* Corresponding author: Xiao-feng Yang

Department of Urology, First Affiliated Hospital of Shanxi Medical University, 85 South Jiefang Road, Taiyuan 030001, China

Tel: 0351-2349145; Fax: 0351-2143253

E-mail: yxfylq@163.com.
}

initial BC, has several shortcomings, while carcinoma in situ is difficult to visualize and distinguish from benign inflammatory lesions ${ }^{[3]}$. WLC is often used to guide TURBt procedures for NMIBC, although it is known to have difficulty in diagnosing papillary lesions. Inadequate visualization of all tumors that may be present, or of diffuse tumor borders, may result in missed or incompletely resected lesions ${ }^{[4]}$. Thus, the search for new imaging technologies has focused on enhancing visualization of bladder tumors to improve diagnostic accuracy. New optical imaging technologies that use different light wavelengths have been used successfully in clinical settings, including fluorescence cystoscopy/ photodynamic diagnosis (PDD), narrow band imaging (NBI), confocal laser endomicroscopy (CLE), and optical coherence tomography (OCT) $]^{[5]}$.

Autofluorescence cystoscopes use light with a wavelength of $308 \mathrm{~nm}^{[6]}$. Fluorescence cystoscopy with intravesical instillation of 5-aminolevulinic acid (5-ALA) uses a $406.7 \mathrm{~nm}$ krypton atom laser transmitter or a xenon lamp filter; the wavelength of the excitation light 

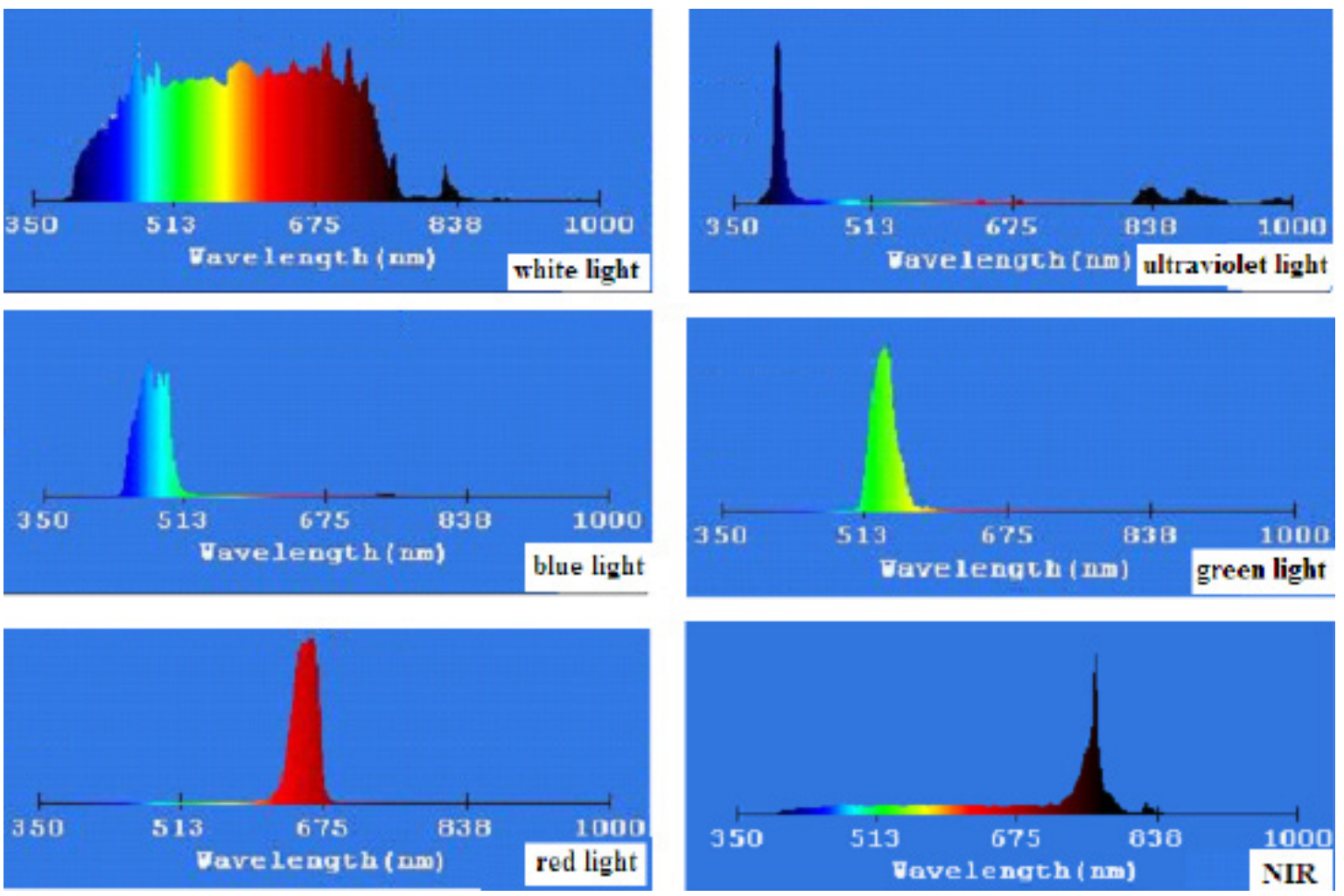

Figure 1. The spectrum of multispectral endoscope light source

is $375-440 \mathrm{~nm}^{[7]}$. The light source of CLE is an argon ion laser with an excitation wavelength of $488 \mathrm{~nm}$. OCT endomicroscopy uses near-infrared light with a wavelength of $800-860 \mathrm{~nm}^{[8]}, 1050 \mathrm{~nm}$, or $1310 \mathrm{~nm}^{[9,10]}$, while NBI cystoscopy uses lights with wavelengths of $415 \mathrm{~nm}$ and $540 \mathrm{~nm}^{[11]}$. Monochromatic or NBI imaging with different wavelengths plays an increasingly important role in cystoscopic optical detection. Therefore, it is necessary to investigate the optical anatomical features of normal bladder mucosa and tumors under a wide spectral range.

Thus, we designed a multispectral endoscope light source that outputs white, ultraviolet, blue, green, red, and near-infrared light, and can be connected through a fiber to a cystoscope for the bladder examination. In this research, we report the imaging characteristics and clinical significance of cystoscopy with a multispectral light source.

\section{MATERIALS AND METHODS}

Patient dates

All 35 patients who received treatment in our hospital were enrolled from May 2010 to May 2013 All patients received multispectral cystoscope sessions, including 14 cases of benign prostatic hyperplasia, 16 cases of non-muscle-invasive bladder tumors, and five cases of patients with upper urinary tract disease and a normal lower urinary tract. Criteria for inclusion in the study were normal urine white blood cell counts, no catheterization or cystoscopy in the previous week, no use of photosensitizer, or benign prostatic hyperplasia without bladder stones. The preoperative clinical stage of BC was determined based on computed tomography images, and a postoperative histopathological examination was performed to determine the pathological stage. The tumors were reviewed according to the classification of 2002 TNM $^{[12]}$.

Approaches with a multispectral cystoscope

The multispectral endoscope light source (Taiyuan SaiEnsi Science and Technology Development Co., Ltd. China), is a new conventional endoscope light source that, when combined with a filter wheel and filters, can output white, ultraviolet, blue, green, red, and near-infrared light. The optical parameter was detected by a HAAS1200 spectrometer (EVERFINE, Hangzhou, China), and is presented in Figure 1. The endoscope and imaging system used an Olympus resectoscope F27, and the Olympus Exera ? CLV-180 camera platform was used in this study. A video line was connected to the S-video output of the CLV-180 camera platform to transfer the dynamic and static images.

After epidural anesthesia, transurethral insertion of the 


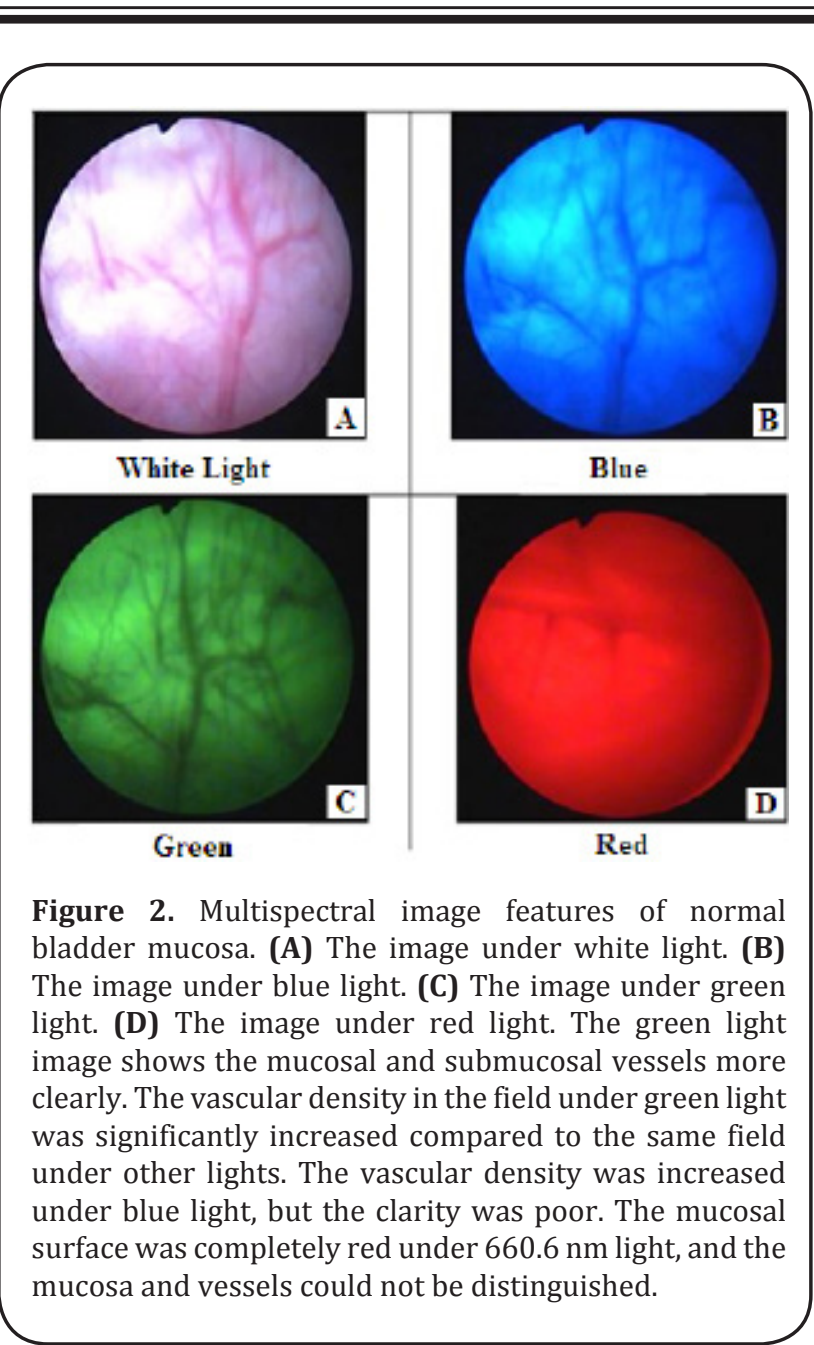

resectoscope was performed, and the bladder was examined under six spectra. Real-time images for the same part of the bladder were acquired, and appropriate treatments were applied accordingly. The biological effects of the different spectra on bladder mucosa were determined based on intravesical visibility, bladder mucosa color, vascular density and clarity, degree of movement between bladder mucosa and submucosa, and the vascular mesh three-dimensional structure of bladder mucosa. Also, the bladder tumor and excision site of TURBt were repeatedly examined.

\section{Statistical analysis}

According to the subjective and objective image analysis methods, semi-quantifications were performed for the images of bladder mucosa from 35 study subjects. The intravesical visibilities were white light $(+++)$, blue and green light (++) and red ( \pm ); bladder mucosa colors were normal, blue, green, and red; vascular density and clarity were white $(++)$, blue $(+)$, green $(+++)$, and red $(-)$. Statistical significance was considered at $\mathrm{p}$ 0 0.05, and all tests were chi-square tests. Statistical analysis was done with $\mathrm{SPSS} \AA$ for Windows $₫$, version 13.0.

\section{RESULTS}
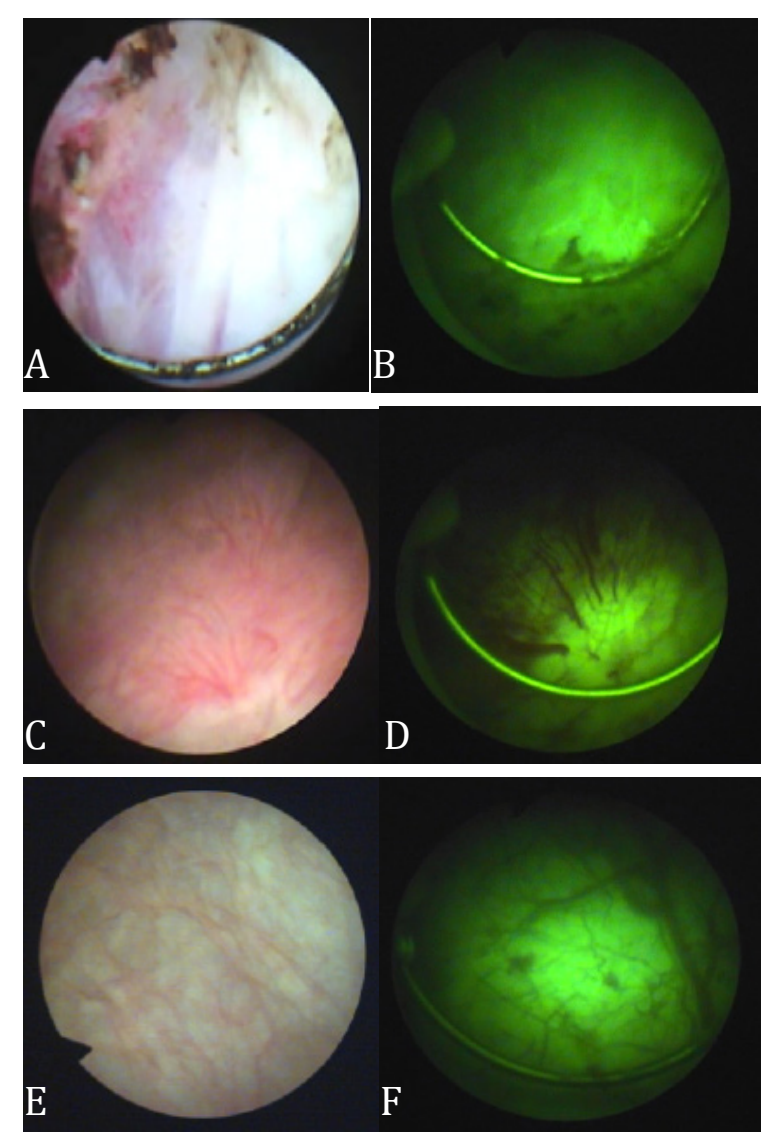

Figure 3. Multispectral image features of BC. (A) Tumor base after TURBt and its edge under white light; (B) The same region under green light, with a uniform color, indicating no blood vessels. (C) and (D) The stage Ta tumor adheres to the mucosal surface only via a small, thin vessel connecting with mucosal blood vessels. The mucosal structure is intact, and the electroexcision loop can thus push the tumor tissue completely away from the mucosal surface. After pushing the tumor tissue away from the mucosa, the white light image shows only spotlike bleeding; however, the green light image not only displays the dark brown ejecting blood flow but also the vascular morphology and the relationship between bleeding spot and mucosal blood vessels. (E) and (F) The stage Tis tumor under green light, as evidenced by the disordered vascular structure and focus on abnormal morphology.

Multispectral image features of normal bladder mucosa

A total of 35 patients received multispectral cystoscopy, including four women and 31 men. Under ultraviolet light and near-infrared light, visibility in the bladder was poor, and the physiological or pathological anatomical structurescould not be seen. Under other lights, the images were clear, as shown in Figure 1.

The best mucosal clarity was obtained when white light was used (Figure 2A). Green light shows the mucosal and submucosal vessels more clearly (Figure 2C). The vascular density in the field under green light was signif- 
icantly increased when compared to the same field under other lights $(\mathrm{P}<0.05)$. Submucosal vessels, together with mucosal vessels, formed a three-dimensional mesh structure as a vascular network floating in the air. When the mucosal surface was pushed, the vascular network rotated back and forth, and the mucosal or submucosal tissues appeared to be absent, leading to a visible muscle surface. Under white light, pushing the mucosal surface also resulted in the dislocation of mucosal and submucosal tissues, but the vascular mesh structure was not evident (Video 1). The vascular density was increased under blue light, but the clarity was poor (Figure 2B). The mucosal surface was completely red under red light, and the mucosa and vessels could not be distinguished (Figure 2D).

\section{Multispectral image features of bladder cancer}

Sixteen cases of stage Tis-T1 NMIBC patients received multispectral imaging examination before TURBt, and the results showed that tumors appeared in different colors under different spectral conditions. Only the images taken under white and green lights had instructive significance for tumor diagnosis and treatment. For small tumors, the white light image could display the morphology of the tumor; the green light image clearly showed the relationship between the tumor and bladder mucosal blood vessels.

For T1 tumors, especially the larger, pedunculated, or sessile ones, multispectral imaging could barely observe the tumor base from the surface and could not distinguish tumors from mucosal blood vessels. When TURBt resection of the tumor reached the submucosa or superficial muscle in the absence of tumor invasion, the integrated structure of superficial muscle was observed under white light but blood vessels were not observed under green light (Figures 3A and 3B).

After completing the excision of visible tumors, the bladder mucosa and other mucosa were further inspected under white and green lights, respectively. The results showed that three cases displayed normal bladder mucosa under white light but showed blood vessels with spot-like structural disorder under green light (Figures $3 \mathrm{E}$ and $3 \mathrm{~F}$ ). These patients were pathologically diagnosed with Tis tumors.

\section{DISCUSSION}

In recent years, optical technology such as cystoscopes and resectoscopes have been significantly improved, leading to the creation of many new tools, including rigid cystoscopes, fiber cystoscopes, autofluorescence cystoscopes, NBI cystoscopes, and CLE. In addition to white light, the light sources of these cystoscopes also include multi-wavelength spectra, such as UV, visible, and near-infrared lights. According to the principles of bio- medical photonics, spectra of different wavelength have different biological effects ${ }^{[13-15]}$. Combined with the characteristics of cystoscopy, we believe that, under the lights of different wavelengths, anatomical images of bladder mucosa differ in various clinical diagnostic values.

Wei et al. studied the penetration depth of light in the human colon and colon adenocarcinoma tissues, showing that light with a wavelength of $890 \mathrm{~nm}$ provided the maximum depth in penetrating the mucosa and submucosa of normal colon and colon adenocarcinoma $(0.517$ and $0.348 \mathrm{~cm}$, respectively), while light with a wavelength of $680 \mathrm{~nm}$ displayed the minimum depth of optical penetration $(0.232 \text { and } 0.209 \mathrm{~cm} \text {, respectively })^{[16]}$. In this study, the penetration depths into the bladder wall were 0.17 and $0.24 \mathrm{~cm}$ for 415 and $540 \mathrm{~nm}$ lights, respectively ${ }^{[17]}$. Ultrasound examination determined that the thickness of the normal bladder wall was 0.1 and $0.3 \mathrm{~cm}$ for full and empty bladders, respectively, while the thickness of bladder wall in patients with dysuria was 0.5-1.2 cm, indicating that light at a certain wavelength can penetrate the bladder wall into the muscle ${ }^{[18]}$.

The physical phenomena of light propagation in biological tissues include reflection, refraction, scattering, and absorption, and the wavelength of light is a very important parameter ${ }^{[19]}$. In the bladder wall, the propagation of light is related not only to the structures of bladder mucosa, submucosa, lamina propria, and muscle tissue but also to the optical absorption of hemoglobin in the blood vessels of the bladder wall.

In the present study, we conducted cystoscopy under six UV-visible-near-infrared spectra, using an Olympus Exera II CLV-180 imaging platform and camera lens. However, this imaging system is not sensitive to UV or near-infrared lights, which is the main cause of a blank monitor screen without any imaging signals. The images shown in Figure 2 were taken under white, blue, green, and red lights, respectively, which suggests that, under green light, the density of blood vessels in the bladder wall increased significantly ( $\mathrm{P}<0.05)$. In particular, the blood vessels in the submucosa were clearly visible; therefore, the blood vessels in both the mucosa and submucosa constituted a three-dimensional network, as if floating in air. When the mucosal surface was pushed, the vascular network rotated back and forth; the mucosal and submucosal tissues were almost invisible, and the muscular surface could be observed directly. This optical phenomenon of the bladder wall will facilitate future indepth investigation of bladder mucosa, mucosal vessels, and their related diseases.

We believe that the basic principle of the formation in this optical phenomenon of the bladder wall is related to the absorption of green spectra by hemoglobin in the blood vessels of the bladder wall. Hemoglobin binds to 02 or $\mathrm{CO} 2$ to form oxyhemoglobin or carboxyhemoglobin, 
respectively. In different structures, hemoglobin molecules absorb lights at different wavelengths and hence show different absorption spectra as the lights transmit through various hemoglobin solutions. Oxyhemoglobin has three characteristic absorption peaks at 415, 541, and $576 \mathrm{~nm}$, respectively. When oxyhemoglobin is converted to carboxyhemoglobin, its absorption spectra also change, displaying three characteristic absorption peaks at 419,540 , and $569 \mathrm{~nm}$, respectively ${ }^{[20]}$. The peak wavelength of the green light in the present study and its penetration depth was about $0.24 \mathrm{~cm}$. Therefore, it was possible to observe the mucosa and submucosa, and even the superficial muscle, under green light. The hemoglobin in the mucosal blood vessels absorbed green light at a wavelength of $541 \mathrm{~nm}$, resulting in dark brown blood vessels in the bladder mucosa and tumor in the imaging, significantly different from the green color in the avascular zones of mucosa and submucosa. The penetration of light through the mucosa of the avascular zone forms the basic principle of the three-dimensional appearance of the superficial blood vessels in bladder mucosa and submucosa. Meanwhile, only part of the blue light was absorbed by hemoglobin, resulting in increased vascular density but a blurred image. Under red light, the mucosal surface appeared all red. Therefore, in the absence of fluorescent dye, blue, and red lights have no clinical significance for cystoscopic diagnosis and treatment.

Based on the above principles of optical imaging of the bladder wall, this study explored the diagnosis and treatment of $\mathrm{BC}$ and found that, under green light, the bases of larger, pedunculated, or sessile T1 tumors were difficult to observe from the tumor surface, and the relationship between mucosal and tumor blood vessels could not be distinguished. As shown in Figure 3, when TURBt resection of the tumor reaches the submucosa or superficial muscle in the absence of tumor invasion, the integrated structure of superficial muscle was observed under white light but blood vessels were not visible under green light. It was thus difficult to distinguish whether there were tumor residues at the wound. For Ta tumors, an electroexcision loop can be used to push the tumor tissue away from the mucosal surface because the tumors are small with a simple structure and are only adhered to the mucosal surface via a thin blood vessel connected to mucosal blood vessels, and the mucosal structure remains intact. An electroexcision loop can be used to push the tumor tissue completely away from the mucosal surface; after pushing the tumor tissue away, WLI of the mucosal surfaceshowed only spot-like bleeding, but green light not only displayed the dark brown ejecting blood flow but also the vascular morphology and the relationship between the bleeding spot and mucosal blood vessels. Also, another finding of the present study was that lesions showing normal mucosa under white light, but showing spot-like disorders of the vascular structure under green light, might be early lesions of bladder or Tis-stage tumors, indicating that green light has some clinical significance in diagnosis and treatment of bladder tumor. However, this diagnosis and treatment technology applies only to the identification of smaller tumors, especially those with a single capillary, and also to the evaluation of the depth of tumor invasion, based on the relationship between tumor vessels and mucosal and submucosal blood vessels.

\section{CONCLUSIONS}

Without photosensitizer or fluorescent dye, tumor blood vessels and bladder mucosal vascular can be seen under green light, following the principle of imaging as related to light absorption by hemoglobin. According to the principle, small tumors and invasive tumor depth can be identified under green light cystoscopy. The new technique shows no diagnostic values for relatively larger tumors, and thus is not necessary in those cases.

\section{CONFLICT OF INTEREST}

The authors declare no conflict of interests.

\section{REFERENCES}

1. Freeman, J. A., Esrig, D., Stein, J. P., Simoneau, A. R., Skinner, E. C., Chen, S. C., Groshen, S., Lieskovsky, G., Boyd, S. D., and Skinner, D. G. (1995) Radical cystectomy for high risk patients with superficial bladder cancer in the era of orthotopic urinary reconstruction. Cancer 76, 833-839

2. Botteman, M. F., Pashos, C. L., Redaelli, A., Laskin, B., and Hauser, R. (2003) The health economics of bladder cancer: a comprehensive review of the published literature. Pharmacoeconomics 21, 1315-1330

3. Babjuk, M., Burger, M., Zigeuner, R., Shariat, S. F., van Rhijn, B. W., Comperat, E., Sylvester, R. J., Kaasinen, E., Bohle, A., Palou Redorta, J., Roupret, M., and European Association of, U. (2013) EAU guidelines on non-muscle-invasive urothelial carcinoma of the bladder: update 2013. Eur Urol 64, 639-653

4. B, G., M, J., R, M., D, G., and P, G. (2010) HAL blue-light cystoscopy in high-risk nonmuscle-invasive bladder cancer--re-TURBT recurrence rates in a prospective, randomized study. Urology 76, 664-669

5. Liu, J. J., Droller, M. J., and Liao, J. C. (2012) New optical imaging technologies for bladder cancer: considerations and perspectives. J Urol 188, 361-368

6. Zaak, D., Stepp, H., Baumgartner, R.,Schneede, P., Waidelich, R., Frimberger, D., Hartmann, A., Kunchel, R., Hofstetter, A., and Hohla, A. (2002) Ultraviolet-excited (308 nm) autofluorescence for bladder cancer detection. Urology 60, 1029-1033

7. Kriegmair, M., Stepp, H., Steinbach, P., Lumper, W., Ehsan, 
A., Stepp, H. G., Rick, K., Knuchel, R., Baumgartner, R., and Hofstetter, A. (1995) Fluorescence cystoscopy following intravesical instillation of 5-aminolevulinic acid: a new procedure with high sensitivity for detection of hardly visible urothelial neoplasias. Urol Int 55, 190-196

8. Keane, P. A., Ruiz-Garcia, H., and Sadda, S. R. (2011) Clinical applications of long-wavelength (1,000-nm) optical coherence tomography. Ophthalmic Surg Lasers Imaging 42 Suppl, S67-74

9. Puvanathasan, P., Forbes, P., Ren, Z., Malchow, D., Boyd, S., and Bizheva, K. (2008) High-speed, high-resolution Fourier-domain optical coherence tomography system for retinal imaging in the $1060 \mathrm{~nm}$ wavelength region. Opt Lett 33, 2479-2481

10. Radhakrishnan, S., Rollins, A. M., Roth, J. E., Yazdanfar, S., Westphal, V., Bardenstein, D. S., and Izatt, J. A. (2001) Real-time optical coherence tomography of the anterior segment at $1310 \mathrm{~nm}$. Arch Ophthalmol 119, 1179-1185

11. Asge Technology, C., Song, L. M., Adler, D. G., Conway, J. D., Diehl, D. L., Farraye, F. A., Kantsevoy, S. V., Kwon, R., Mamula, P., Rodriguez, B., Shah, R. J., and Tierney, W. M. (2008) Narrow band imaging and multiband imaging. Gastrointest Endosc 67, 581-589

12. Li, X., Dong, M., Lin, Q., Chen, Z. H., Ma, X. K., Xing, Y. F., Wan, X. B., Wen, J. Y., Wei, L., Chen, J., and Wu, X. Y. (2013) Comparison of current staging systems for advanced hepatocellular carcinoma not amendable to locoregional therapy as inclusion criteria for clinical trials. Asia Pac J Clin Oncol 9, 86-92

13. Ren, H., Waltzer, W. C., Bhalla, R., Liu, J., Yuan, Z., Lee, C. S., Darras, F., Schulsinger, D., Adler, H. L., Kim, J., Mishail,
A., and Pan, Y. (2009) Diagnosis of bladder cancer with microelectromechanical systems-based cystoscopic optical coherence tomography. Urology 74, 1351-1357

14. Sonn, G. A., Mach, K. E., Jensen, K., Hsiung, P. L., Jones, S. N., Contag, C. H., Wang, T. D., and Liao, J. C. (2009) Fibered confocal microscopy of bladder tumors: an ex vivo study. J Endourol 23, 197-201

15. Banerjee, S., and Sharma, S. K. (2010) Use of Monte Carlo simulations for propagation of light in biomedical tissues. Appl Opt 49, 4152-4159

16. Jiang, W. H., Da, X., Hua, H. B., Min, G. H., Guangzhou, Guangzhou, and Guangzhou. (2005) Optical Penetration Depths and Light Attenuation Characteristics of Normal and Adenomatous Human Colon Tissue in Vitro. Chinese Journal of Lasers

17. Naselli, A., Introini, C., Timossi, L., Spina, B., Fontana, V., Pezzi, R., Germinale, F., Bertolotto, F., and Puppo, P. (2012) A randomized prospective trial to assess the impact of transurethral resection in narrow band imaging modality on non-muscle-invasive bladder cancer recurrence. Eur Urol 61, 908-913

18. Xue-Su, Y. U., J-Jiong, M. A., and Zhang, Z. (2008) Clinical value and ultrasound diagnosis in bladder trabecula and cellula. Journal of Medical Imaging

19. Zhang, Z. X. (2008) Town Biomedical Photonics and application of new technology. Beijing: Science and Technology Press, 10.

20. Ruan, P., Huang, Y. X., and Li, D. (2005) [Spectrum analysis of erythrocyte intracellular hemoglobin with a novel fast multi-channel micro-spectrophotometry]. Guang Pu Xue Yu Guang Pu Fen Xi 25, 1121-1124 